\title{
Exercise training as therapy for chronic heart failure
}

\author{
Neal G Uren, David P Lipkin
}

Patients with impaired left ventricular function have a reduced exercise capacity because of dyspnoea and fatigue; and exercise testing has become an integral part of the assessment of patients with chronic heart failure in many cardiac centres. ${ }^{1}$ Over the past decade, several groups have shown that there is also a clinical benefit from using regular exercise testing and training as adjunctive therapy in the treatment of selected patients with chronic heart failure. The underlying mechanisms responsible for the beneficial effect of exercise training are now becoming apparent.

\section{Normal cardiovascular response to exercise}

During isotonic exercise the contraction of skeletal muscle in movement provides a volume rather than a pressure load to the left ventricle, and the cardiovascular response is proportional to the severity of the exercise. ${ }^{2}$ The human body responds to dynamic exercise so that the blood flow to exercising muscles is increased to maintain oxygen delivery, heat is dissipated, and flow to vital organs such as the brain and heart is preserved. The cardiac output, the oxygen capacity of the blood (dependent on arterial oxygen saturation and haemoglobin concentration), and the vascular bed of exercising and non-exercising skeletal muscle all function concordantly to ensure that oxygen delivery approximates to oxygen demand. The intensity of exercise may be quantified by the maximal oxygen consumption (the product of maximum cardiac output and maximum arteriovenous oxygen $\left(\mathrm{a}-\mathrm{v} \mathrm{O}_{2}\right)$ difference) which is the greatest amount of oxygen an individual can use during dynamic exercise to deliver to skeletal muscle. ${ }^{3}$ Because the $a-v$ $\mathrm{O}_{2}$ difference has a physiological limit, at true maximal effort maximal oxygen consumption is an estimate of maximal cardiac output. ${ }^{2}$

The normal response to exercise is an increase in cardiac output with an overall reduction in systemic vascular resistance caused by vasodilatation in exercising muscle and a subsequent small increase in arterial blood pressure. ${ }^{4}$ Other peripheral adaptations include the central displacement of venous blood through muscle contraction of the exercising limbs and the abdominal and thoracic muscles. The cardiac response to exercise occurs through the integrated effects of tachycardia, increased adrenergic stimulation, and the Frank-Starling mechanism. ${ }^{4}$ In mild to moderate exercise the heart rate parallels increasing cardiac output mainly through loss of vagal restraint. With more strenous exercise adrenergic activation supplements this exercise tachycardia and increases myocardial contractility, leading to an increased stroke volume (with an increase in end diastolic volume through the Frank-Starling mechanism). Other adaptations improving peripheral oxygen delivery are an increased oxygen extraction in exercising muscles and the rightward shift of the oxygen-haemoglobin dissociation curve. ${ }^{4}$ With regular training, healthy people may develop a lower heart rate at rest and on exercise. In trained athletes additional ventricular hypertrophy and dilatation facilitate a higher and more sustained cardiac output on exercise.

\section{Cardiovascular response to exercise in} chronic heart failure

In the early stages of chronic heart failure compensatory mechanisms maintain cardiac output. These are an increase in end-diastolic volume with increased preload (the FrankStarling mechanism), left ventricular hypertrophy, and the increased neurohormonal response. As heart failure progresses, however, an inability to increase adequately cardiac output in response to exercise develops, with an additional decrease in skeletal muscle perfusion at rest and on exercise associated with an increased vascular resistance in exercising muscle. ${ }^{5}$ Although cardiac output can be redistributed through vasoconstriction so that blood flow to exercising muscles and vital organs is conserved, this compensatory response may become defective. As a consequence arterial blood pressure is maintained on exercise to preserve flow to non-exercising vascular beds to the detriment of blood flow to exercising muscle at rest and on exercise. ${ }^{5}$ Maximal oxygen extraction in exercising muscle is reached more quickly than in healthy individuals and there is greater reliance on anaerobic metabolism. The reduction in blood flow to the exercising skeletal muscle, and thus in oxygen delivery, relates closely to the reduced peak cardiac output and exercise capacity in these patients. ${ }^{5-7}$

Skeletal muscle abnormalities in chronic heart failure

In chronic heart failure, either through the reduction in cardiac output or physical decon- 
ditioning, the vasoreactivity of the skeletal muscle vascular bed becomes impaired and the oxidative capacity is altered. Several histological and histochemical changes have been noted in the skeletal muscle of patients with heart failure. In different studies, atrophy of type I fibres ${ }^{8-10}$ and type II fibres ${ }^{8112}$ or an increased percentage of type IIb fibres ${ }^{10} 12$ have been reported. These resemble the changes seen after deconditioning in healthy people.

A reduction in blood flow to exercising limbs during submaximal and maximal exertion was an early peripheral abnormality found in chronic heart failure. ${ }^{13}$ Further work showed that this failure to augment maximal blood flow adequately in exercising muscle may occur independently of cardiac performance on exercise. ${ }^{14}$ Maximal blood flow to exercising skeletal muscle is reduced in chronic heart failure to some extent as a result of a normal mean arterial blood pressure being maintained. ${ }^{5}$ This reduced flow results from a reduced muscle driving pressure (arterial minus venous pressure) as well as impaired vasodilatation in the muscle bed. ${ }^{1314}$ However, at a given work rate, forearm muscle blood flow was similar in patients with heart failure and in healthy controls, ${ }^{15}$ implying that blood flow matches workload in a small muscle group but that this linear relation does not hold in larger muscle groups where vasodilatation is impaired.

Magnetic resonance spectroscopy of high energy phosphate metabolism ( $\left.{ }^{31} \mathrm{P}-\mathrm{MRS}\right)$ showed excessive dependence on anaerobic glycolysis with rapid depletion of phosphocreatine and early lactic acid production in the forearm muscles of patients with chronic heart failure compared with healthy controls. ${ }^{16-18}$ This excessive depletion of phosphocreatine on exercise occurs to a greater extent in exercising leg muscles in the absence of any difference in leg blood flow at the same workload. ${ }^{15} \mathrm{Al}$ though Massie et al found a correlation between such skeletal muscle metabolic abnormalities in the forearm on exercise and the clinical severity of chronic heart failure, the skeletal muscle abnormalities were unrelated to muscle blood flow. ${ }^{16}$ Thus the reduced exercise capacity of chronic heart failure cannot be explained by changes in muscle blood flow alone.

It is likely that there is a defect of oxidative metabolism in skeletal muscle in chronic heart failure, which is consistent with the reduction in volume of mitochondrial cristae in skeletal muscle cells. ${ }^{19}$ Although concentrations of glycolytic enzymes are reported to be normal, ${ }^{10} 12$ mitochondrial enzymes involved in substrate use such as succinate dehydrogenase and $\beta$-hydroxyacyl-coenzyme $\mathrm{A}$ dehydrogenase are reduced. ${ }^{10}$ However, it has been difficult to correlate early phosphocreatine depletion with such histochemical abnormalities. ${ }^{12}$

\section{Exercise training as therapy}

Formerly, exercise training in patients with chronic heart failure was believed to be harmful, causing worsening of symptoms and further compromising cardiac function. Though there is some evidence to suggest that exercise training may cause further changes in left ventricular wall thinning and shape distortion in patients with a large degree of ventricular asynergy soon after myocardial infarction, ${ }^{20}$ there is no evidence for functional deterioration in patients with well compensated chronic heart failure. ${ }^{21}$ Several studies over the past decade showed that exercise programmes were not harmful but were of benefit when carefully controlled protocols were used. ${ }^{22-25}$ Exercise capacity measured as maximal oxygen consumption or exercise duration may be increased and this increase is associated with several beneficial peripheral and metabolic adaptations..$^{22-25}$ The remaining uncertainties about exercise as a treatment relate to patient selection and to the optimal intensity and duration of therapy.

Lee et al first showed improvement in mean exercise duration after long-term physical training in 18 patients with chronic heart failure who showed no change in resting cardiac output or central haemodynamic variables. $^{22}$ In another study to investigate the central and peripheral haemodynamic and metabolic adaptations to long-term exercise, Sullivan et al studied 12 patients (mean (SEM) left ventricular ejection fraction of $24(10) \%$ ) before and four to six months after four hours of regulated exercise per week and showed a mean increase of $23 \%$ in maximal oxygen consumption. ${ }^{23}$ There was also a reduction in heart rate at rest and submaximal exercise and an increase in the systemic a $-\mathrm{v} \mathrm{O}_{2}$ difference at rest and peak exercise, which was first noted after 75 days of training. There were no changes at either rest or exercise in arterial pressure, pulmonary wedge pressure, stroke volume, or ejection fraction, with only a trend towards an increase in cardiac output. The increase in the systemic a $-\mathrm{vO}_{2}$ difference was attributed to a redistribution of cardiac output to working skeletal muscles. In addition to this central adaptation there were increases in leg blood flow (with reductions in vascular resistance) and delivery of oxygen to the leg at peak exercise, in a $-\mathrm{v}_{2}$ difference (greater oxygen extraction) and a reduction in lactate production at submaximal exercise. The fall in lactate production was independent of leg blood flow, implying a true peripheral adaptation independent of haemodynamic changes. There was also a significant increase in haemoglobin concentration after training. The response to exercise was unrelated to any resting variable: the change in maximal oxygen consumption correlated with the increase in cardiac output and leg blood flow and also with the amount of time spent exercising each week. ${ }^{23}$ In another study of patients in New York Heart Association class I who followed a more intensive training regimen, Ehsani showed increases both in stroke volume and in maximal peripheral oxygen extraction. ${ }^{24}$

It has been suggested that some of the secondary changes seen in chronic heart failure-such as neurohormonal activation and changes in skeletal muscle and peripheral vasculature-may be a result of physical decon- 
ditioning itself. The autonomic imbalance (increased sympathetic activity) seen in moderate to severe chronic heart failure may reduce heart rate variability and thus predispose the patient to sudden death..$^{25}$ In one study, 11 patients with stable moderate to severe heart failure (left ventricular ejection fraction $19(8) \%$ ) performed 20 minutes of daily bicycle exercise (five days a week) to 60 $80 \%$ maximum heart rate over eight weeks in a crossover study with eight weeks rest. Exercise duration and maximal oxygen consumption increased by around $20 \%$. This increase in maximal oxygen consumption correlated closely with a reduced heart rate at rest and submaximal exercise, an increased heart rate variability, and a reduced resting noradrenaline spillover, suggesting an association between the improvement in exercise capacity and reduced autonomic imbalance. ${ }^{25}$

\section{Mechanisms for improvement in exercise capacity with training}

The mechanism whereby the low output state of chronic heart failure alters the oxidative capacity of skeletal muscle is not known. Possible mechanisms include increased activity of the sympathetic nervous system or neurohormonal changes or chronic low grade ischaemia The metabolic changes found in the finger flexor muscles of patients in heart failure do not support disuse as a cause in its own right. ${ }^{16}$ Musch et al studied the effects of low and high intensity exercise training in rats with chronic heart failure. ${ }^{26}$ High intensity training conferred an additional increase in the maximal $\mathrm{a}-\mathrm{v} \mathrm{O}_{2}$ difference, a reduction in resting and maximal heart rate (reversal of "chronotropic incompetence") and an increase in cardiac output compared with low intensity training. Whether the extent of exercise performed in humans determines the extent of changes in skeletal muscle blood flow and metabolism is not known. Other possible adaptations accounting for the training-induced delay in anaerobic metabolism, in addition to vascular and biochemical adaptations in skeletal muscle, are a decreased adrenergic activity with exercise, ${ }^{25}$ changes in substrate delivery, or alterations in intramuscular blood flow distribution. ${ }^{15}$ The increase in haemoglobin concentration (which could account for increased peripheral oxygen delivery) probably occurs after training because diuretic treatment prevents an increase of plasma volume. ${ }^{23}$

Peripheral deconditioning accounts for the impairment of vasodilator capacity to a large degree. Sinoway et al showed that a unilateral programme of dynamic handgrip over 30 days led to a greater peak blood flow response to ischaemia and lower minimal vascular resistance in the trained forearm of patients with heart failure (compared with the control forearm), in the absence of changes in systemic aerobic capacity. ${ }^{27}$ Skeletal muscle blood flow and metabolism are closely linked because the distribution of blood flow on exercise is matched to local metabolism. It is likely that the benefits of physical training also occur predominately through reversal of the reduc- tion in oxidative capacity. In another study of the independent peripheral adaptation to regular exercise, Minotti et al performed a localised training regimen on the forearm of patients with heart failure. Endurance improved and was associated with a reduction in the ratio of inorganic phosphate to phosphocreatine $\left(\mathrm{P}_{\mathrm{i}} / \mathrm{PCr}\right.$ ) at ${ }^{31} \mathrm{P}-\mathrm{MRS}$ (indirect evidence of increased oxidative capacity). ${ }^{28}$ This occurred in the absence of changes in forearm blood flow, which implies that regulated exercise improves the metabolic response of skeletal muscle to exercise. It may be that this is a major mechanism whereby exercise training improves exercise capacity in chronic heart failure.

It is also of interest that improvement in maximal oxygen consumption may lag behind objective measurements of resting cardiac function after the start of drug treatment; this is further evidence of a coupling process between cardiac performance and circulatory and metabolic function during exercise in patients with chronic heart failure. ${ }^{29}$

\section{Conclusions}

Exercise training benefits patients with wellcompensated chronic heart failure, even in those with considerable left ventricular impairment. It delays the onset of anaerobic metabolism in skeletal muscle reversing the impairment of peripheral vasodilatation and improving blood flow to exercising muscles. It is safe and does not induce clinical deterioration as previously believed. However, many previous studies have not taken patient motivation and thus compliance into account. More controlled studies are required to determine the intensity and duration of exercise training required. It is likely that those who show no adaptation to a low intensity training programme within six weeks should not continue with the programme. ${ }^{21}$ The use of exercise training as a treatment in patients with chronic heart failure has provided another means of treating a pathophysiological state that otherwise has a natural history of gradual deterioration and high mortality despite early pharmacological intervention. However, whether this increase in exercise capacity can be sustained and reflected in an improved survival is not known

1 Patterson JA, Naughton J, Pietras RJ, Gunnes RM. Treadmill exercise in the assessment of the functional capacity of mill exercise in the assessment of the functional capacity of patients

2 Fletcher GF, Froelicher VF, Hartley $H$, Haskell WL Pollock ML. Exercise standards. A statement for health professionals from the American Heart Association. Cir culation 1990;82:2286-332.

3 Weber KT, Kinasewitz GT, Janicki JS, Fishman AP. Oxygen utilization and ventilation during exercise in patients with chronic heart failure. Circulation 1982;65:1213-23.

4 Braunwald E, Ross J. Control of cardiac performance. In Berne RM, Sperelakis N, Geiger SR, eds. The cardiovascular system. Vol 1: The heart. Baltimore: Waverly Press, 1979:550-63.

5 Sullivan MJ, Knight JD, Higginbotham MB, Cobb FR Relation between central and peripheral hemodynamics during exercise in patients with chronic heart failure. Circulation 1989;80:769-81.

6 Franciosa JA, Leddy CL, Wilen M, Schwartz DE. Relation between hemodynamic and ventilatory responses in determining exercise capacity in severe congestive heart failure. Am J Cardiol 1984;53:127-34.

7 Wilson JR, Me 1984;53:127-34. DE, Ferraro N. Exercise intolerance in patients with chronic heart failure: role of 
impaired nutritive flow to skeletal muscle. Circulation 1984;69:1079-87.

8 Lipkin DP, Jones DA, Round JM, Poole-Wilson PA. Abnormalities of skeletal muscle in patients with chronic heart failure. Int J Cardiol 1988;18:187-95.

9 Caforio ALP, Rossi B, Risaliti R, Sciliano G, Marchetti A, Angelini $C$ et al. Type I fiber abnormalities in skeletal muscle of patients with hypertrophic and dilated cardiomyopathy: evidence of subclinical myogenic myopathy. J Am Coll Cardiol 1989;14:1464-73.

10 Sullivan MJ, Green HJ, Cobb FR. Skeletal muscle biochemistry and histology in ambulatory patients with longterm heart failure. Circulation 1990;81:518-27.

11 Dunnigan A, Staley NA, Smith SA, Pierpont ME, Judd D, Benditt DG et al. Cardiac and skeletal muscle abnormalities in cardiomyopathy: comparison of patients with malities in cardiomyopathy: comparison of patients with ventricular tachycardia or conge
Coll Cardiol 1987;10:608-18.

12 Mancini DM, Coyle E, Coggan A. Contribution of intrinsic Mancini DM, Coyle E, Coggan A. Contribution of intrinsic
skeletal muscle changes to ${ }^{31} \mathrm{P}$ NMR skeletal muscle skeletal muscle changes to
metabolic abnormalities in patients with chronic heart metabolic abnormalities in patients
failure. Circulation 1989;80:1338-46.

13 Zelis R, Longhurst J, Capone RJ, Mason DT. A comparison of regional blood flow and oxygen utilisation during dynamic forearm exercise in normal subjects and patients with congestive heart failure. Circulation 1974;50: 137-43.

14 Lejemtel TH, Maskin CS, Lucido D, Chadwick BJ. Failure to augment maximal limb blood flow in response to oneleg versus two-leg exercise in patients with severe heart failure. Circulation 1986;74:245-51.

15 Arnolda L, Conway M, Dolecki M, Sharif H, Rajagopalan B, Ledingham JGG et al. Skeletal muscle metabolism in heart failure: $a{ }^{31} \mathrm{P}$ nuclear magnetic resonance spectroscopy study of leg muscle. Clin Sci 1990;79:583-9.

16 Massie BM, Conway M, Yonge R, Frostick S, Ledingham J, Sleight $P$ et al. Skeletal muscle metabolism in patients with congestive heart failure: relation to clinical severity and congestive heart failure: relation to clinical
blood flow. Circulation 1987;76:1009-19.

17 Weiner DH, Fink LI, Maris J, Jones RA, Chance B, Wilson JR. Abnormal skeletal muscle bioenergetics during exercise in patients with heart failure: role of reduced muscle blood flow. Circulation 1986;73:1127-36.

18 Massie BM, Conway M, Rajagopalan B, Yonge R, Frostick S, Ledingham J et al. Skeletal muscle metabolism during
exercise under ischaemic conditions in congestive heart exercise under ischaemic conditions in congestive heart
failure. Evidence for abnormalities unrelated to blood failure. Evidence for abnormal

19 Drexler H, Riede U, Schaefer H-E. Reduced oxidative capacity of skeletal muscle in patients with severe heart failure. Circulation 1987;76 (suppl IV):IV-178.

20 Jugdutt BI, Michorowski BL, Kappagoda CT. Exercise training after anterior $Q$ wave myocardial infarction importance of regional left ventricular function and topography. J Am Coll Cardiol 1988;12:362-72.

21 Kellermann JJ, Shemesh J, Ben-Ari E. Contraindications to physical training in patients with impaired ventricular function. Eur Heart $J$ 1988;9 (suppl F):71-6.

22 Lee AP, Ice R, Blessey R, Sanmarco ME. Long-term effects of physical training on coronary patients with impaired LV function. Circulation 1979;60:1519-26.

23 Sullivan MJ, Higginbotham MB, Cobb FR. Exercise training in patients with severe left ventricular dysfunction: ing in patients with severe left ventricular dysfunction: 78:506-15.

24 Ehsani AA. Adaptations to training in patients with exerciseinduced left ventricular dysfunction. Adv Cardiol 1986; 34:148-55.

25 Coats AJS, Adamopoulos S, Meyer T, Conway J, Sleight P. Physical training in chronic heart failure. Lancet 1990; 335:63-6.

26 Musch TI, Moore RL, Hilty MR. Effects of dynamic exercise training on the metabolic and cardiocirculatory responses to exercise in the rat model of myocardia infarction and heart failure. Am J Cardiol 1988;62: 20E-24E (Suppl).

27 Sinoway LI, Shenberger J, Wilson J, Mclaughlin D, Musch $T$, Zelis R. A 30-day forearm work protocol increases maximal forearm blood flow. I Appl Physiol 1987; 62:1063-7.

28 Minotti JR, Johnson EC, Hudson TL, Zuroske G, Murata $\mathrm{G}$, Fukushima $\mathrm{E}$ et al. Skeletal muscle response to exercise training in congestive heart failure. J Clin Invest training in cong

29 Zelis R, Sinoway LI, Musch TI, Davis D, Just H. Regional blood flow in congestive heart failure: concept of compensatory mechanisms with short and long time constants. $A m$ J Cardiol 1988;62:2E-8E (Suppl). 